Check for updates

Cite this: RSC Adv., 2019, 9, 15431

Received 26th January 2019

Accepted 1st May 2019

DOI: $10.1039 / c 9 r a 00698 b$

rsc.li/rsc-advances

\title{
Investigation of the adsorption behavior of BSA with tethered lipid layer-modified solid-state nanopores in a wide $\mathrm{pH}$ range $\uparrow$
}

\author{
Haibing Tian,,$^{\mathrm{ab}}$ Wanyi Xie, $\mathbb{D} t^{\mathrm{ab}}$ Shixuan He, ${ }^{\mathrm{a}}$ Daming Zhou, ${ }^{\mathrm{a}}$ Shaoxi Fang, ${ }^{\mathrm{a}}$ \\ Liyuan Liang (iD) *ab and Deqiang Wang*ab
}

\begin{abstract}
Nanopore technology was introduced for the study of the dynamic interactions between bovine serum albumin (BSA) and 1,2-dioleoyl-sn-glycero-3-phosphoethanolamine (DOPE) phospholipids based on a modified nanopore. The results reveal that the interaction mechanism between DOPE and BSA is affected by the $\mathrm{pH}$ of the subphase. Far above the BSA isoelectric point $(\mathrm{pH}>7)$, a weaker hydrophobic interaction and stronger electrostatic repulsion exist between the DOPE and BSA molecules. At $\mathrm{pH}=7$, the BSA structure nearly does not change, and the interaction is weak. At $\mathrm{pH} 5$ and $\mathrm{pH} 6, \mathrm{BSA}$ is marginally affected by the adsorption interaction, and below $\mathrm{pH}$, the DOPE film becomes disordered, so there is a strong repulsive force interaction between the BSA and DOPE.
\end{abstract}

\section{Introduction}

In recent years, liposomes have received increasing attention in developing efficient drug and pharmaceutical delivery systems. ${ }^{1}$ 1,2-dioleoyl-sn-glycero-3-phosphorethanolamine (DOPE) is an important component of liposome carriers. DOPE greatly affects the liposomes' activities in vivo due to its neutral property. ${ }^{2}$ Therefore, numerous research studies have focused on the interaction between plasma constituents and liposomes in order to improve the bioavailability of drugs. In the last few years, researchers have developed a variety of protein-lipid interaction characterization methods based on surface measurements, such as surface pressure, electrical potential and microscopic visualization measurements. ${ }^{3-7}$ However, all of the aforementioned methods are limited for the study of the interplay between lipids and proteins in situ. Nanopore technology is a promising approach for the study of DNA unzipping kinetics, DNA-protein interactions, and the dynamics of proteins. $^{8-17}$ The investigation of the dynamics of these biopolymers is based on their interactions with the nanopore walls. Bovine serum albumin (BSA) is one of the main proteins in bovine serum; it belongs to the globular proteins and has great conformational adaptability. Therefore, in this work, we introduce nanopore technology for the study of the interaction between BSA and DOPE.

${ }^{a}$ Chongqing Institute of Green and Intelligent Technology, Chinese Academy of Science, Chongqing 400714, P. R. China. E-mail: dqwang@cigit.ac.cn

${ }^{b}$ University of Chinese Academy of Sciences, P. R. China

$\dagger$ Electronic supplementary information (ESI) available. See DOI: 10.1039/c9ra00698b

$\$$ These authors contributed equally to this paper.
To date, there are a variety of approaches using direct physisorption of lipids onto solid supports. ${ }^{18,19}$ However, the interface between the lipid membrane and solid substrate is not well-controlled, and the lifetime of non-covalently attached lipids is limited. ${ }^{20}$ In this report, we firstly develop a simple feasible bottom-up approach to assemble a tethered lipid layer (TLL) on the nanopore surface, and the modification steps are shown in Scheme 1a (left). The applied nanopores were fabricated via electric pulse breakdown. The organosilane GOPS directly self-assembles on the nanopore surface to form an epoxy group-coated layer for further reaction. The terminal epoxy groups were used to covalently attach the amino group on DOPE via an epoxy-amine reaction to form the TLL modified $\operatorname{SiN}_{x}$ nanopore, as shown in Scheme $1 \mathrm{~b}$.

\section{Results and discussion}

The film hydrophilicity, thickness, and chemical composition for each modification step of the $\operatorname{SiN}_{x}$ substrate were characterized with contact angle measurements and X-ray photoelectron spectroscopy (XPS). As shown in Scheme 1a (right), the film hydrophilicity apparently changed after each modification treatment for the substrate. The contact angle of the uncoated $\operatorname{SiN}_{x}$ nanopore was found to be $36.42^{\circ}$ after Piranha treatment, while it was $69.62^{\circ}$ after the modification with DOPE. This suggests that the introduction of a lipid layer on the substrate contributes to the increase of the hydrophobicity of the film. XPS measurements were performed to verify the chemical composition of the coated film (Fig. S1†). In Table S1, $\dagger$ the element percentages of the bare (Piranha-treated) $\mathrm{SiN}_{x}$, GOPScoated film, and DOPE film are compared. The $\operatorname{SiN}_{x}$ exhibits strong signals for $\mathrm{O}$ after coating with GOPS, which was 
(a)

\section{SiN Membrane}
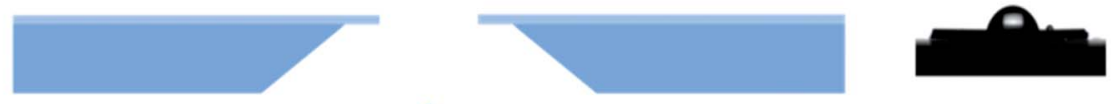

$69.62^{\circ}$

Piranha solution, $70^{\circ} \mathrm{C}, 30 \mathrm{~min}$
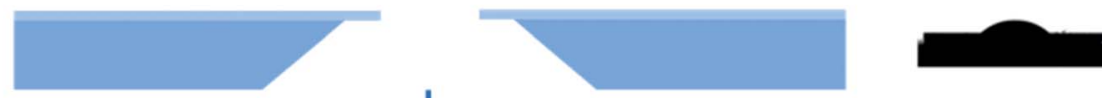

$36.42^{\circ}$

$-\downarrow$ GOPS, r.t. 30min
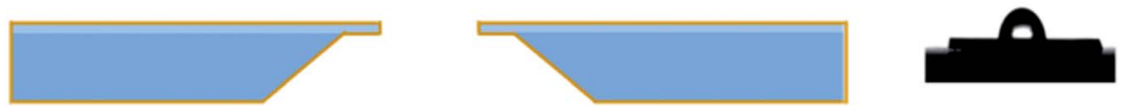

$83.36^{\circ}$

\section{DOPE, r.t. $6 h$}
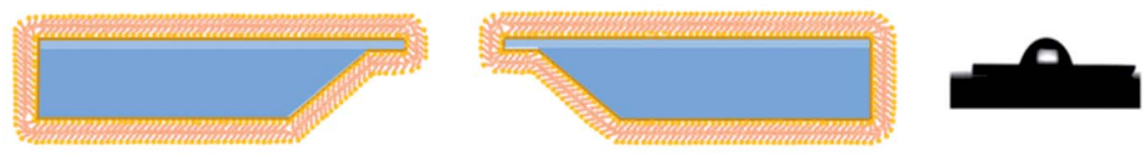

$70.34^{\circ}$

(b)
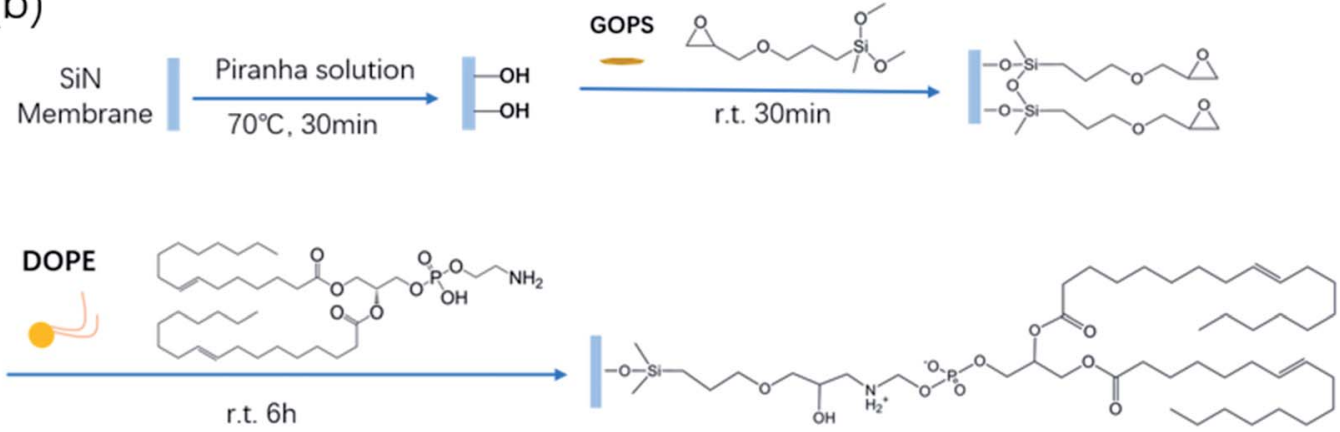

Scheme 1 (a) Schematic of the chemical modification steps of the $\mathrm{SiN}_{x}$ nanopore with DOPE (left), and the film hydrophilicity test (right). (b) The reaction mechanism of the chemical modification.

attributed to the terminal epoxy group, and the DOPE film displays a high signal for $\mathrm{C}$, which arises from the alkyl groups.

However, these characterization techniques described above cannot be used to probe the coating inside a nanopore. The ion flux through the nanopores is extremely sensitive to the nanopore coating thickness, since the ionic conductance $(G)$ depends quantitatively on the nanopore diameter $(d)$.

The effective diameter was estimated based on the empirical formula: ${ }^{21,22}$

$$
G=\sigma\left[\left(4 L / \pi d^{2}\right)+(1 / d)\right]^{-1}
$$

Herein, $\sigma$ is the electrical conductivity $(1 \mathrm{M} \mathrm{KCl}, \mathrm{pH} 7,10.6 \mathrm{~S}$ $\mathrm{m}^{-1}$ ), and $L$ is the length of the nanopore. In order to testify this, the ion-conductance of the bare and modified nanopores was measured (Fig. 1a and b) on a patch clamp. In terms of the size of the TLB modified nanopore, $d^{\prime}=d_{\text {bare }}-2 \delta$, where $d_{\text {bare }}$ is the calculated diameter of the uncoated nanopore and $\delta$ is the thickness of the DOPE film. According to a literature report, the thickness of the DOPE layer is about $3.6 \mathrm{~nm} .{ }^{18,23}$ The length of the modified nanopore is increased by the lipid layer coating, $L^{\prime}=L_{\text {bare }}+2 \delta$, where $L_{\text {bare }}$ is the $\operatorname{SiN}_{x}$ thickness $(20 \mathrm{~nm}$ in this work). The calculated diameters of the bare $\operatorname{SiN}_{x}$ nanopore and the TLL-modified nanopore are $20 \mathrm{~nm}$ and $13 \mathrm{~nm}$ respectively, which agrees very well with the physical sizes measured by the TEM technique (Fig. 1c and d).

Moreover, the ionic current noise between the bare nanopore and the TLL-modified nanopore was compared. The power spectrum densities (PSDs) were obtained from the 10 second baseline. As shown in Fig. 2a, the $1 / f$ noise has been depressed after the modification, which is favorable for recording the translocation signature. In addition, the $I-V$ curves were recorded to testify the stability of the TLL nanopore with a broad range of $\mathrm{pH}$ values (from 3 to 10) in $1 \mathrm{M} \mathrm{KCl} \mathrm{(Fig.} \mathrm{2).}$

This result indicates that the conductance of the TLLmodified nanopore does not change significantly under these conditions (Fig. 2, inset). In order to explore the potential applications of the protein adsorption interaction using the 
(a)

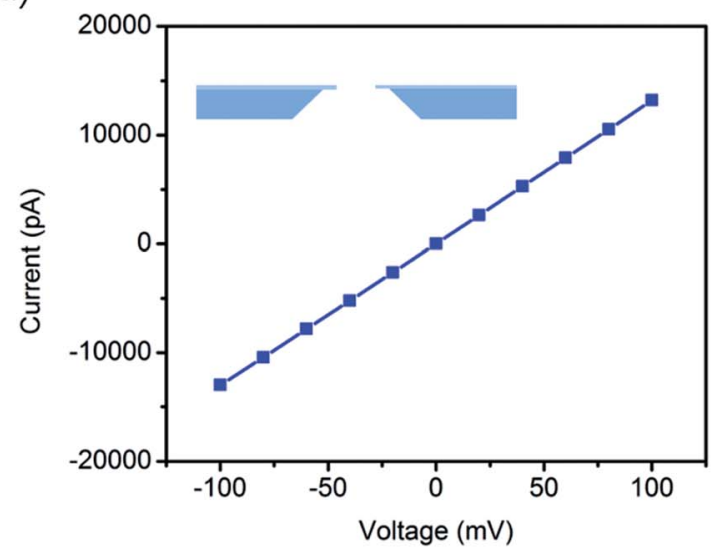

(c)

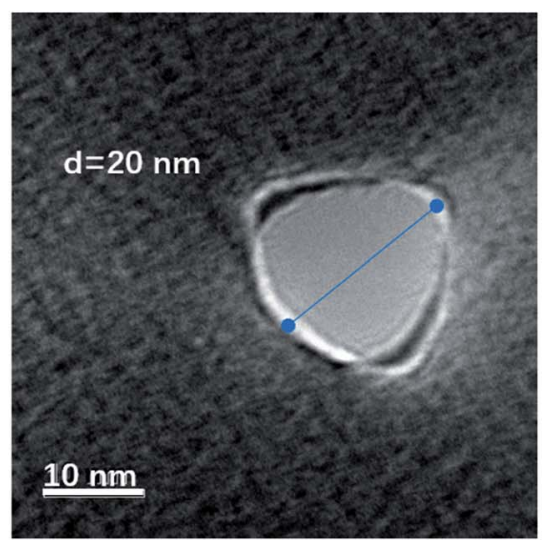

(b)

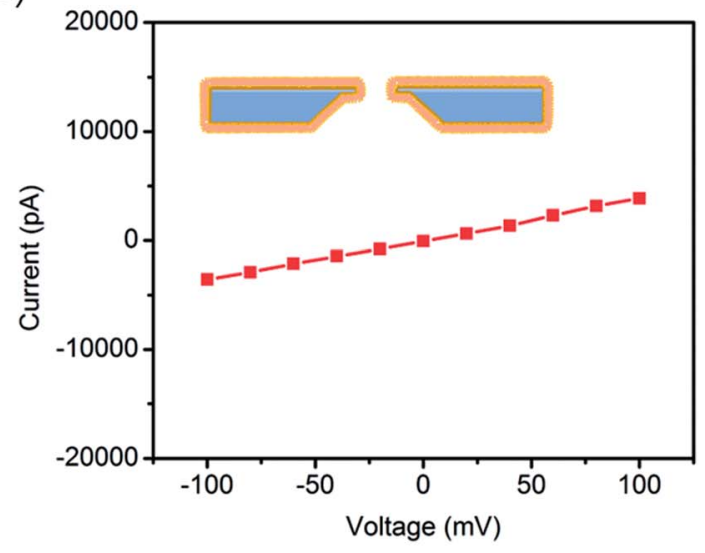

(d)

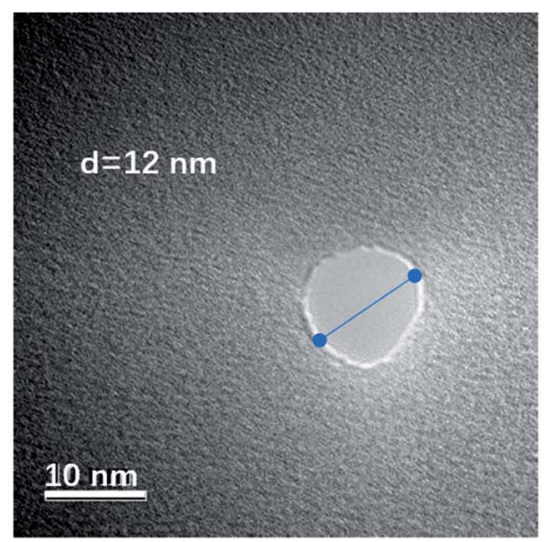

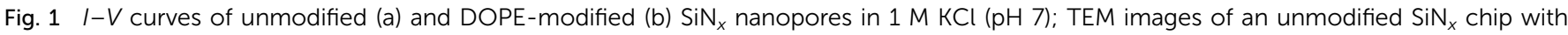
a nanopore of $20 \mathrm{~nm}$ diameter (c) and a DOPE-modified $\mathrm{SiN}_{x}$ chip with a nanopore of $12 \mathrm{~nm}$ diameter (d).

TLL-modified nanopore, we carried out measurements at distinct $\mathrm{pH}$ values for BSA. The ionic current traces of $0.5 \mu \mathrm{M}$ BSA translocation were recorded in $1 \mathrm{M} \mathrm{KCl}(10 \mathrm{mM}$ Tris, $1 \mathrm{mM}$ EDTA) buffer at various $\mathrm{pH}$ values with a modified nanopore of $13 \mathrm{~nm}$ diameter at $100 \mathrm{mV}$.
According to the reported data, the isoelectric point (PI) of BSA ranges from $\mathrm{pH} 5.1$ to $\mathrm{pH} 5.5 .{ }^{15}$ Hence, BSA is negativelycharged above $\mathrm{pH} 5$, and electrically-neutral at $\mathrm{pH}$. The cumulative ionic current trace results are presented in event distribution plots (Fig. 3). The ionic current was measured at $\mathrm{pH}$ (a)

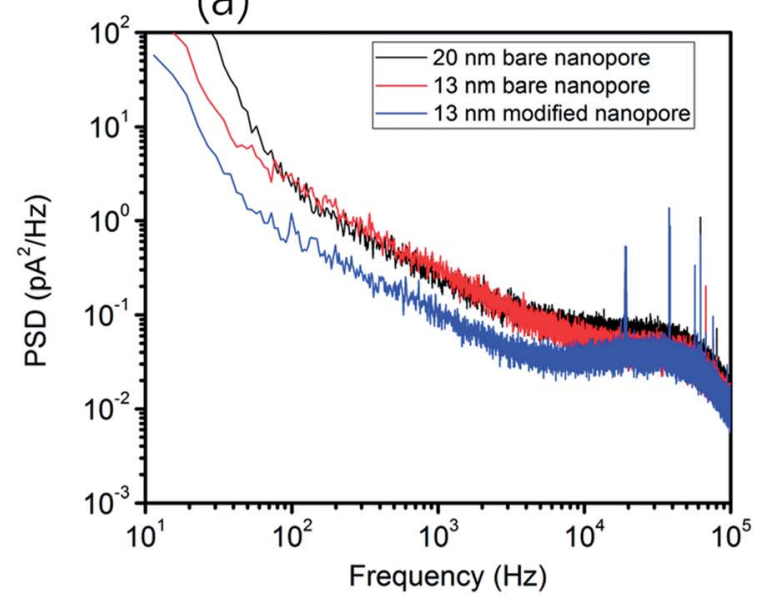

(b)

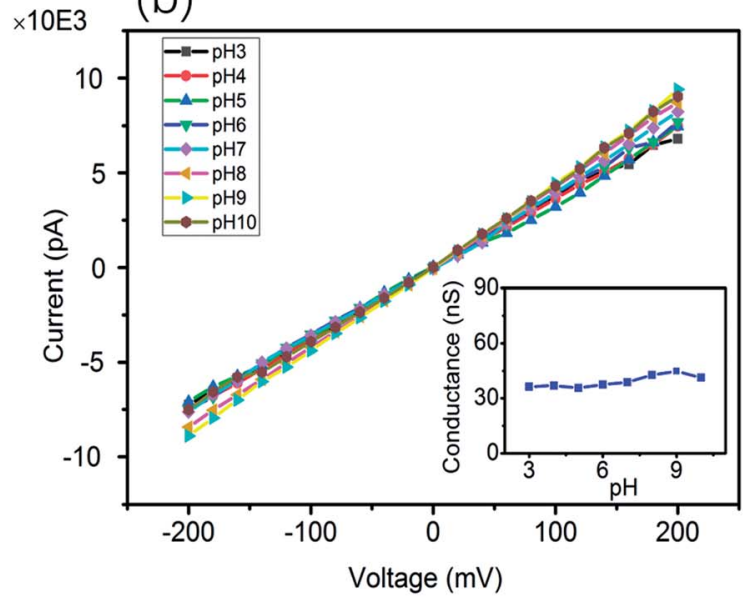

Fig. 2 (a) Power spectrum densities (PSDs) of the ionic current baseline for the bare nanopore of 20 nm diameter (black), the bare nanopore of $13 \mathrm{~nm}$ diameter (red) and the TLL-modified (blue) nanopore of $13 \mathrm{~nm}$ diameter at $100 \mathrm{mV}$ with $1 \mathrm{M} \mathrm{KCl}(\mathrm{pH}$ 7). (b) I-V curves of the TLL-modified nanopore of $13 \mathrm{~nm}$ diameter at various $\mathrm{pH}$ values (from 3 to 10) in $1 \mathrm{M} \mathrm{KCl}$ buffer solution; the inset is the nanopore conductance as a function of $\mathrm{pH}$ for the modified nanopore. 
(a)
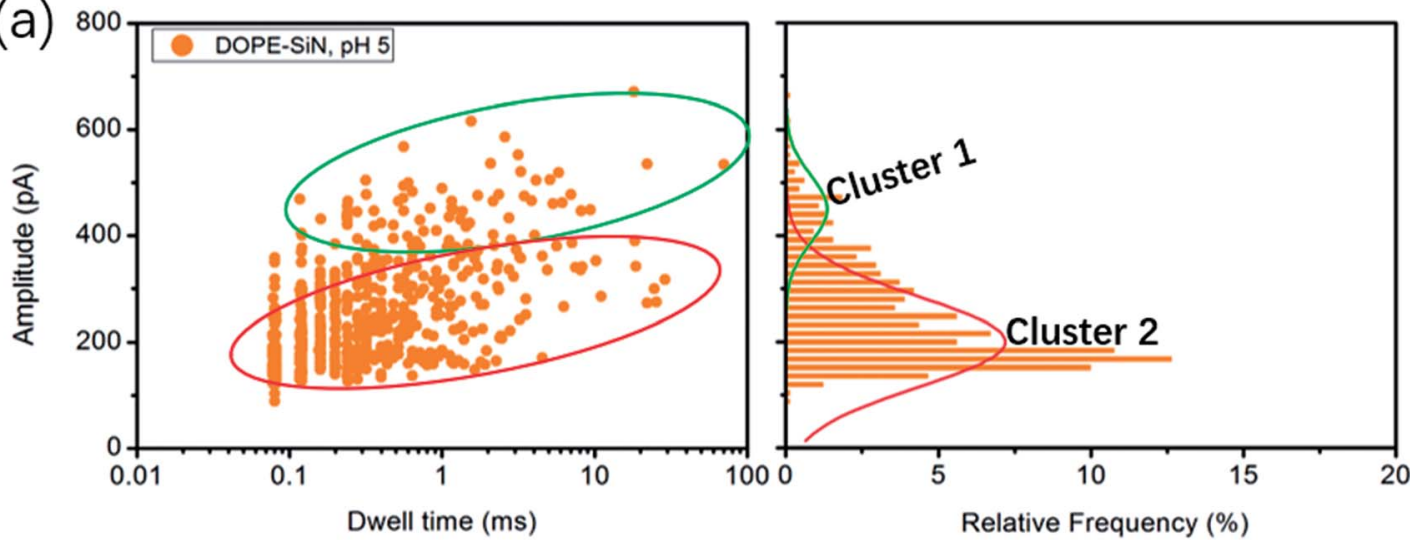

(b)
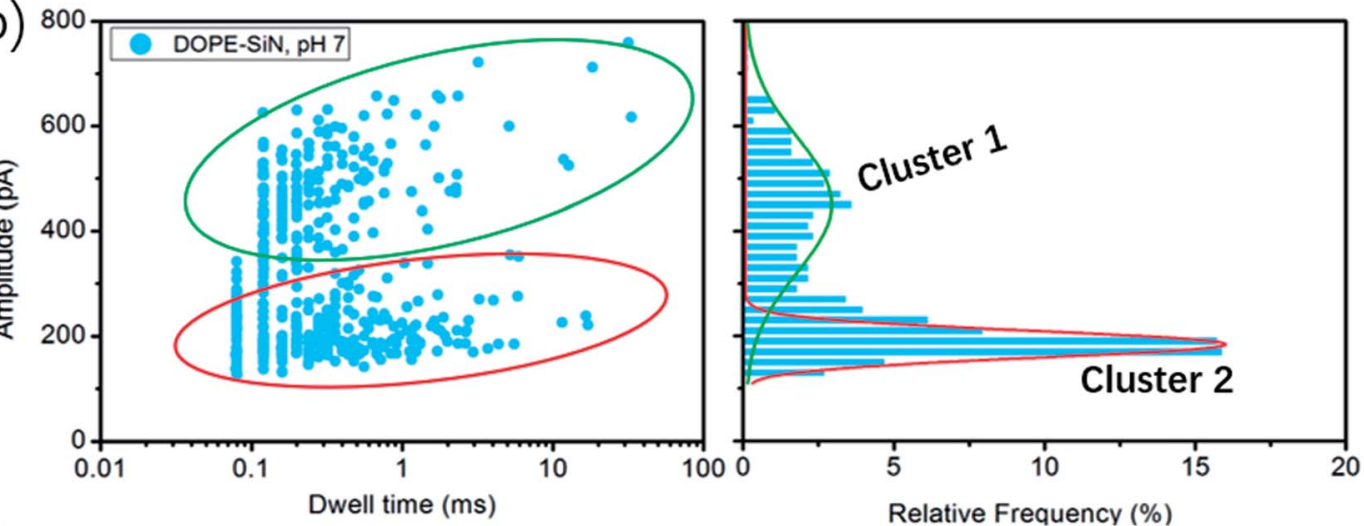

(c)
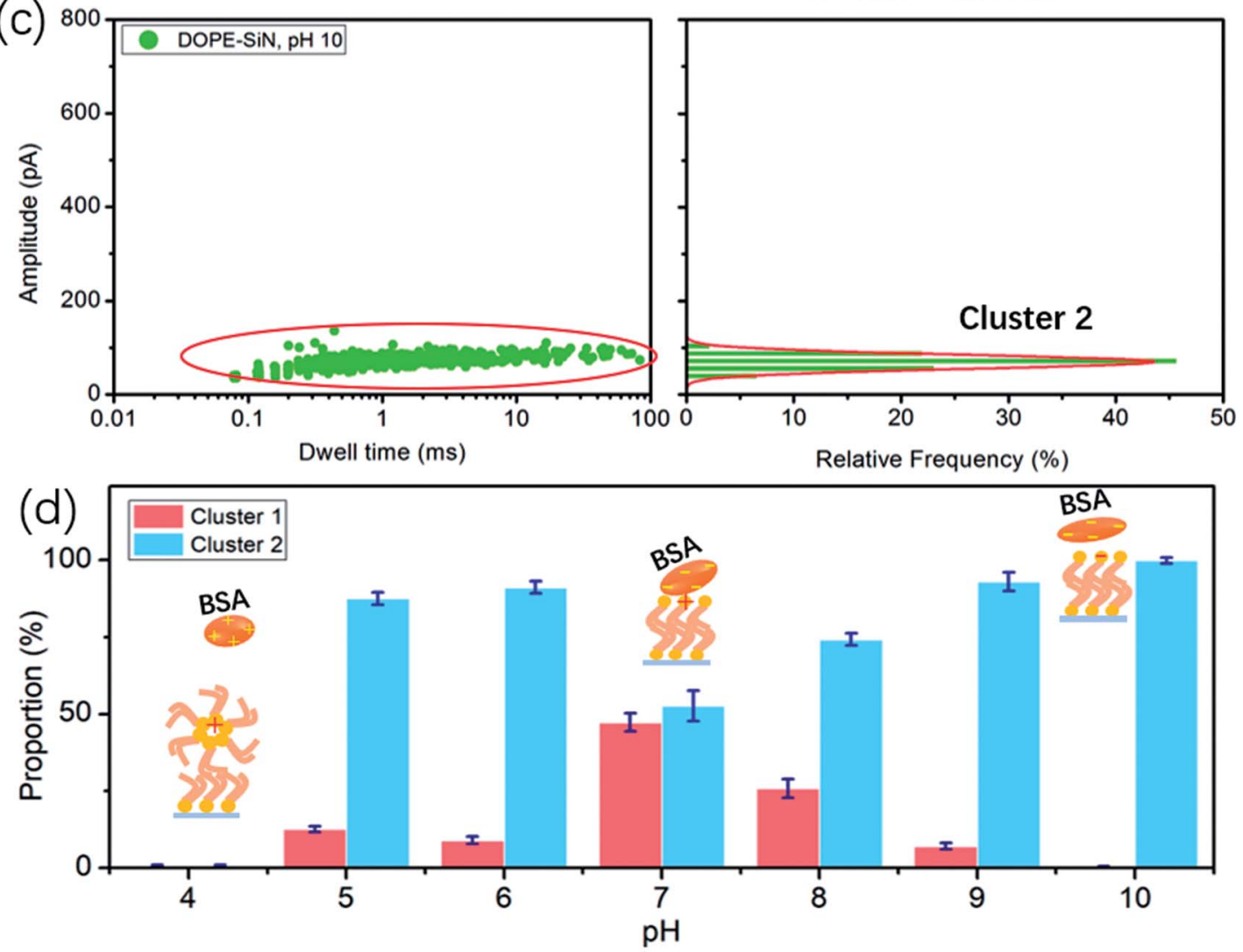

Fig. 3 Event scatter plots for the translocation of $0.5 \mu \mathrm{M}$ BSA through a $13 \mathrm{~nm}$ TLL-modified nanopore under $100 \mathrm{mV}$ at pH 5 (a), $\mathrm{pH} 7$ (b) and $\mathrm{pH} 10$ (c). Amplitude histograms are fitted with Gaussian distributions. (d) The proportion of cluster 1 and cluster 2 at different pH values (from 4-10). 
5 , which is close to the isoelectric point of BSA. Fig. 3a shows that there are two clusters of BSA blockage events: the current drop $(\Delta I)$ of cluster 1 is mainly between 400 and $600 \mathrm{pA}$, and the $\Delta I$ of cluster 2 is $100-300 \mathrm{pA}$. At $\mathrm{pH} 7$, there are also two clusters (Fig. 3b). Compared with the results at $\mathrm{pH} 5$, the percentage of cluster 2 became higher and the blockage time became shorter (Fig. 3d).

Previous studies have demonstrated that the current drop is approximately proportional to the excluded volume $(V)$ of the translocating particle inside the nanopore and can be approximately described as: $:^{24}$

$$
\Delta I=\left(\sigma \psi / L_{\mathrm{eff}}^{2}\right) V
$$

In this formula, $\sigma$ is the conductivity of the solution $(1 \mathrm{M} \mathrm{KCl}$, $\left.\mathrm{pH} 7,10.6 \mathrm{~S} \mathrm{~m}^{-1}\right), \psi$ is the applied voltage, and $L_{\text {eff }}$ is the effective length of the nanopore ( $\sim 27 \mathrm{~nm}$ in this work). It is reported that the volume of BSA is about $224 \mathrm{~nm}^{3}$. Therefore, according to formula (2), the calculated $\Delta I$ of BSA in this experiment is $326 \mathrm{pA}$. Previous studies have shown that to compare long thin rods with spheres and short rods of the same volume, for the spheres and short rods $\Delta I$ must be multiplied by $3 / 2$ for the values to agree on an absolute basis. ${ }^{25}$ Thus, the value of $\Delta I$ after absolute calibration is close to $490 \mathrm{pA}$. This suggests that cluster 1 events are attributed to BSA molecules translocating through the nanopores, and cluster 2 events are protein molecules that failed to pass through. At pH 5 the electric force of BSA in the nanopore is lower than that of BSA at $\mathrm{pH} 7$, which causes more molecules to fail to pass through the nanopore at pH 5.

Earlier research shows that the volume of BSA molecules is expanded at low pH values. ${ }^{26}$ Based on formula (2), $\Delta I$ increases with the increase of the volume. However, we couldn't get any current blockage signal below $\mathrm{pH}$ 5. According to a previous study, ${ }^{27}$ DOPE lipid easily forms a hexagonal (HII) phase at $\mathrm{pH}$ values below 5 . The hexagonal phase is considered to exhibit a 'cone' shape, where the polar headgroup region is at the smaller end of the cone. ${ }^{24}$ The alkyl groups of DOPE are exposed outside, and the DOPE film and BSA become positively-charged at $\mathrm{pH}$ values below 5 . As a result, the repulsive fore between BSA and the disordered DOPE film hinders BSA from moving to the interface, such that it is hard to get a nanopore translocation event.

At $\mathrm{pH} \mathrm{10,} \mathrm{far} \mathrm{away} \mathrm{from} \mathrm{PI,} \mathrm{there} \mathrm{is} \mathrm{only} \mathrm{one} \mathrm{cluster,} \mathrm{as}$ presented in Fig. 3c, and the current drop becomes very short (only around $100 \mathrm{pA}$ ), but the blockage time is obviously longer than that under neutral and acidic solution conditions. That is to say, barely any BSA proteins successfully translocate through the TLL-modified nanopore. Exposure to alkali will cause BSA dimerization, unfolding, and eventual aggregation. The hydrophobic interaction between BSA and DOPE becomes weak due to the few hydrophobic groups of BSA become exposed. DOPE is gradually changed to negatively-charged, and from $\mathrm{pH} 8$ to 10 the proportion of cluster 1 becomes less and less (Fig. S2a and $\mathrm{b} \dagger)$. This illustrates that, in alkali solution, the electrostatic repulsion between BSA and DOPE becomes the main interaction, which influences the BSA translocation through the modified nanopore.

\section{Experimental}

\section{Materials and methods}

Materials. The $\operatorname{SiN}_{x}$ membranes were purchased from Norcada (NT001Y), with a thickness of $20 \mathrm{~nm}$ and a window size of $10 \mu \mathrm{m} \times 10 \mu \mathrm{m}$. Anhydrous methanol was acquired from Aldrich. 3-Glycidyloxypropyltrimethoxysilane (GOPS), 1,2-dioleoyl-sn-glycero-3-phosphoethanolamine (DOPE), potassium chloride (KCl), ethylenediaminetetraacetic acid (EDTA) and bovine serum albumin (BSA) were purchased from Sigma. Deionized water was obtained from a molecular $\mathrm{H}_{2} \mathrm{O}$ system. The buffer solution was filtered with a $0.22 \mu \mathrm{m}$ filter before use.

Ionic transport through the nanopore. The prepared nanopore chip was embedded in a two-chamber cell where both sides of the nanopore were separated. Each side was filled with $1 \mathrm{M}$ $\mathrm{KCl}, 10 \mathrm{mM}$ Tris- $\mathrm{HCl}$, and $1 \mathrm{mM}$ EDTA buffer at different $\mathrm{pH}$ values (from $\mathrm{pH} 3$ to $\mathrm{pH} 10$ ). Two $\mathrm{Ag} / \mathrm{AgCl}$ electrodes were inserted into each chamber. A bias voltage was applied by a patch clamp amplifier (Axon 200B). The ion current blockade measurements were performed at 8-pole and $40 \mathrm{kHz}$ with pClamp 10 software. All of the demonstrated events in the figures have been filtered by a $10 \mathrm{kHz}$ low-pass Bessel filter for clarity.

BSA translocation through the nanopore. The prepared nanopore chip was embedded in a two-chamber cell where both sides of the nanopore were separated. Each side was filled with $1 \mathrm{M} \mathrm{KCl}, 10 \mathrm{mM}$ Tris- $\mathrm{HCl}$, and $1 \mathrm{mM}$ EDTA buffer at different $\mathrm{pH}$ values (from $\mathrm{pH} 5$ to $\mathrm{pH}$ 10). BSA solution was diluted to 0.5 $\mu \mathrm{M}$. Two Ag/AgCl electrodes were inserted into each chamber. A bias voltage was applied by a patch clamp amplifier (Axon 200B). The ion current blockade measurements were performed at 8-pole and $40 \mathrm{kHz}$ with pClamp 10 software. All of the demonstrated events in the figures have been filtered by a 10 $\mathrm{kHz}$ low-pass Bessel filter for clarity.

\section{Nanopore fabrication and modification}

The nanopores were fabricated with $\mathrm{SiN}_{x}$ membranes (thickness $20 \mathrm{~nm}$ ) via electric pulse breakdown, which was performed on Keithley 2450 equipment. The diameter of the obtained nanopores is about $20 \mathrm{~nm}$. First, the freshly prepared nanopores were treated with Piranha solution (volume ratio $1: 3$ $\mathrm{H}_{2} \mathrm{O}_{2}: \mathrm{H}_{2} \mathrm{SO}_{4}$ ) for $30 \mathrm{~min}$ at $70{ }^{\circ} \mathrm{C}$, followed by rinsing with deionized water to clean the nanopores and simultaneously introduce hydroxyl groups onto the interior nanopore surface. Then, the chip was soaked in $0.000005 \%$ GOPS anhydrous methylbenzene solution for $30 \mathrm{~min}$, followed by washing with methylbenzene, acetone, isopropanol, and deionized water to remove the residual GOPS silane on the pore surface. After forming epoxy groups on the surface, the chips were treated with 0.001 M DOPE for 6 hours and then washed with chloroform and alcohol.

\section{Characterization}

Contact angle (DSA100) equipment was used for the detection of the hydrophilicity of the films. A spectroscopic ellipsometer (Gaertner L116) was used to characterize the film thickness. An 
XPS instrument (Escalab 250Xi) equipped with a monochromatic Al-K $\alpha$ source was used to analyze the chemical components of the films.

\section{Conclusions}

In this study, we introduce nanopore technology to investigate the interaction between BSA and lipid films based on a TLLmodified solid-state nanopore. By analyzing BSA translocation at different $\mathrm{pH}$ values, these results reveal that the interaction between DOPE and BSA is affected by the $\mathrm{pH}$, as shown in Fig. 3d.

(i) Far above the PI $(\mathrm{pH}>7)$, a weaker hydrophobic interaction and stronger electrostatic repulsion exist between the DOPE and BSA molecules, so a lower blockage current and longer blockage time for BSA are observed.

(ii) At $\mathrm{pH}=7$, the BSA structure nearly does not change and the highest current is detected.

(iii) BSA carries nearly zero net charge at $\mathrm{pH} 5$ and $\mathrm{pH} 6$ (Fig. S2c $\dagger$ ), and is marginally affected by the adsorption interaction.

(iv) At $\mathrm{pH}$ values below 5, the DOPE film becomes disordered, and no translocation events are observed due to the strong repulsive force interaction between BSA and DOPE.

\section{Conflicts of interest}

There are no conflicts to declare.

\section{Acknowledgements}

We thank the Key Research Program of the Chinese Academy of Sciences (Grant No. KFZD-SW-219), the Natural Science Foundation of Chongqing, China (Grant No. cstc2018jcyjA1467, cstc2017jcyjB0105), the National Natural Science Foundation of China (Grant No. 41603089, 61701474), and the Youth Innovation Promotion Association (2017392) for supporting this work.

\section{Notes and references}

1 G. Caracciolo, D. Pozzi, A. L. Capriotti and C. Chiara, J. Nanopart. Res., 2013, 15, 1498.

2 T. Gjetting, N. S. Arildsen, C. L. Christensen, T. T. Poulsen, J. A. Roth, V. N. Handlos and H. S. Poulsen, Int. J. Nanomed., 2010, 5, 371.

3 R. verger and F. Pattus, Chem. Phys. Lipids, 1982, 30, 189.

4 H. Brockman, Curr. Opin. Struct. Biol., 1999, 9, 438.
5 G. Xu, C. Hao, L. Zhang, S. Chen and R. Sun, Chin. J. Chem. Phys., 2017, 30, 595.

6 J. L. Richens, J. S. Lane, J. P. Bramble and P. O'Shea, Biochim. Biophys. Acta, Biomembr., 2015, 1848, 1828.

7 A. J. García-Sáez and P. Schwille, Methods, 2008, 46, 116.

8 Y. Feng, Y. Zhang, C. Ying, D. Wang and C. Du, Genomics Proteomics Bioinformatics, 2015, 13, 4.

9 L. Q. Gu and J. W. Shim, Analyst, 2010, 135, 441.

10 B. Hornblower, A. Coombs, R. D. Whitaker, A. Kolomeisky, S. J. Picone, A. Meller and M. Akeson, Nat. Methods, 2007, 4, 315 .

11 Q. Zhao, R. S. S. de Zoysa, D. Wang, D. A. Jayawardhana and X. Guan, J. Am. Chem. Soc., 2009, 131, 6324.

12 E. C. Yusko, B. R. Bruhn, O. M. Eggenberger, J. Houghtaling, R. C. Rollings, N. C. Walsh, S. Nandivada, M. Pindrus, A. R. Hall, D. Sept and J. Li, Nat. Nanotechnol., 2017, 12, 360.

13 N. Giamblanco, D. Coglitore, J. M. Janot, P. E. Coulon, B. Charlot and S. Balme, Sens. Actuators, B, 2018, 260, 736.

14 X. Li, R. Hu, J. Li, X. Tong, J. J. Diao, D. Yu and Q. Zhao, Appl. Phys. Lett., 2016, 109, 143105.

15 D. Fologea, B. Ledden, D. S. McNabb and J. Li, Appl. Phys. Lett., 2007, 91, 053901.

16 J. Sha, B. Xu, Y. Chen and Y. Yang, Acta Chim. Sin., 2017, 75, 1121.

17 S. L. Cai, L. X. Zhang, K. Zhang, Y. B. Zheng, Y. B. Zheng, S. Zhao and Y. Q. Li, Microchim. Acta, 2016, 183, 981.

18 E. C. Yusko, J. M. Johnson, S. Majd, P. Prangkio, R. C. Rollings, J. Li, J. Yang and M. Mayer, Nat. Nanotechnol., 2011, 6, 253.

19 B. M. Venkatesan, J. Polans, J. Comer, S. Sridhar, D. Wendell, A. Aksimentiev and R. Bashir, Biomed. Microdevices, 2011, 13, 671.

20 W. Zhou and P. J. Burke, ACS Appl. Mater. Interfaces, 2017, 9, 14618.

21 S. W. Kowalczyk, A. Y. Grosberg, Y. Rabin and C. Dekker, Nanotechnology, 2011, 22, 315101.

22 B. Yin, W. Xie, L. Liang, Y. Deng, S. He, F. He, D. Zhou, C. Tlili and D. Wang, ACS Omega, 2017, 2, 7127.

23 B. A. Lewis and D. M. Engelman, J. Mol. Biol., 1983, 166, 211. 24 D. S. Talaga and J. Li, J. Am. Chem. Soc., 2009, 131, 9287.

25 E. C. Gregg and K. D. Steidley, Biophys. J., 1965, 5, 393.

26 T. Peters Jr, Serum albumin, Adv. Protein Chem., 1985, 37, 161.

27 P. T. Cullis and B. D. Kruijff, Biochim. Biophys. Acta Rev. Biomembr., 1979, 559, 399. 